\title{
Felicidad, emociones positivas, evaluación cognitiva y afrontamiento en estudiantes universitarios de Lima Metropolitana
}

\author{
Happiness, Positive Emotions, Cognitive Appraisal, and \\ Coping in University Students of Metropolitan Lima
}

\author{
José Loayza-Rivas ${ }^{1}$ \\ (1) https://orcid.org/0000-0003-1505-8304
}

'Departamento de Psicología, Universidad Tecnológica del Perú, Perú

\begin{abstract}
Resumen. Objetivo. El objetivo principal fue examinar la influencia de la felicidad, por medio de la evaluación cognitiva y las emociones positivas, en el afrontamiento del estrés en estudiantes universitarios. Método. Participaron 352 personas, $70.2 \%$ mujeres y 29.8\% hombres, con un rango de edad entre 19 y 35 años. Se realizaron modelos de ecuaciones estructurales (SEM, por sus siglas en inglés) para estimar la influencia de la felicidad en el proceso del estrés y el afrontamiento. Resultados. Los resultados mostraron correlaciones esperadas entre las variables y un buen ajuste de los modelos propuestos. La felicidad influye en la utilización de estrategias de afrontamiento adaptativas, a través de la evaluación cognitiva de desafío $(\beta=.220 ; p<.001)$ y la experiencia de emociones positivas $(\beta=.159 ; p=.001$ ). Se concluye que la felicidad tiene un importante papel en el proceso del estrés y el afrontamiento.
\end{abstract}

Palabras clave. Felicidad, emociones positivas, evaluación cognitiva, afrontamiento, estudiantes universitarios.

Abstract. Objective. The main objective of this study was to examine the influence of happiness by cognitive appraisal and positive emotions on stress coping in university students. Method. 352 people participated in the study, $70.2 \%$ were women, with an age range of between 19 and 35 years. Structural Equation Models (SEM) were tested to estimate the influence of happiness in the stress process and coping. Results. The results showed expected correlations between the variables and a good fit of the proposed models. Happiness influences the use of adaptive coping strategies, through the cognitive appraisal of challenge $(\beta=$ .220; $p<.001)$ and the experience of positive emotions $(\beta=.159 ; p=.001)$. In conclusion, happiness plays an important role in the stress and coping process.

Keywords. Happiness, Positive Emotions, Cognitive Appraisal, Coping, University Students.

1José Loayza-Rivas. Departamento de Psicología, Universidad Tecnológica del Perú. Dirección postal: Avenida El Sol, cuadra 2, Lima 36, Lima, Perú. E-mail: jloayza.89@gmail.com

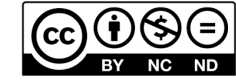

Esta obra está bajo una licencia de Creative Commons Reconocimiento-NoComercial-SinObraDerivada 4.0 Internacional. 


\section{Introducción}

La felicidad puede entenderse como un estado de satisfacción que se experimenta subjetivamente en posesión de un bien deseado (Alarcón, 2006). Implica hacer un juicio de satisfacción sobre la propia vida en términos positivos, por un lado, sentir frecuentemente emociones y estados de ánimo positivos y, por otro, experimentar pocas emociones y estados de ánimo negativos (Diener, 2000). Si bien es cierto, la felicidad suele ser vista como un estado final que resulta del cumplimiento de objetivos deseados, los psicólogos han comenzado a preguntarse si la felicidad puede jugar un rol importante en ayudar a las personas a conseguir tales objetivos (Diener et al., 2018; Lucas \& Diener, 2008).

Este cambio de orientación en la investigación sobre la felicidad ha generado creciente evidencia que muestra que la felicidad puede promover resultados positivos en la vida de las personas (Diener \& Chan, 2011; Diener \& Tay, 2012; Lyubomirsky et al., 2005). Por ejemplo, Lyubomirsky et al. (2005) realizaron un meta-análisis sobre la relación entre felicidad y éxito, encontrando que las personas felices obtienen resultados exitosos en distintos dominios de vida, tales como desempeño laboral, relaciones sociales, ingresos económicos, salud y longevidad. Estos autores concluyeron que el afecto positivo, un importante componente de la felicidad, tiene la capacidad de provocar resultados de éxito y que las personas felices poseen una serie de atributos, recursos y habilidades que les ayudan a prosperar y tener éxito.

Una vía por la cual se puede estudiar si la felicidad promueve un funcionamiento efectivo es explorando su papel en el proceso del estrés y el afrontamiento, puesto que la felicidad afecta la forma en que las personas perciben y evalúan los eventos de vida (Abbe et al., 2003; Lyubomirsky, 2001) y contribuye a superar más rápidamente las situaciones estresantes y adversas (Fredrickson et al., 2003).

Desde una perspectiva transaccional, el estrés es definido como "una relación particular entre el individuo y el entorno que es evaluado por este como amenazante o desbordante de sus recursos y que pone en peligro su bienestar" (Lazarus \& Folkman, 1986, p. 43). En ese sentido, existen dos procesos que tienen lugar en la experiencia subjetiva del estrés: la evaluación cognitiva y el afrontamiento.

Por una parte, la evaluación cognitiva alude a la valoración que se hace de la situación estresante y a las propias habilidades y recursos personales disponibles para manejarla. De acuerdo con el modelo biopsicosocial existen dos tipos generales de evaluación cognitiva: desafío y amenaza (Blascovich, 2008; Tomaka et al., 1993; Tomaka et al., 1997). La evaluación de desafío ocurre cuando la situación es relevante y los recursos personales son suficientes para hacer frente a las demandas de la tarea o de la situación estresante. Mientras que la evaluación de amenaza implica que la situación es relevante, pero los recursos personales son percibidos como insuficientes para hacer frente a las demandas de la tarea o situación estresante (Blascovich, 2008; Tomaka et al., 1993; Tomaka et al., 1997).

Por otro lado, el afrontamiento se refiere a aquellos esfuerzos cognitivos y conductuales para manejar las demandas específicas externas y/o internas de los eventos estresantes. Lazarus y Folkman (1986) plantearon dos funciones del afrontamiento: enfocado en el problema (estrategias dirigidas a cambiar la relación persona-ambiente) y enfocado en la emoción (estrategias dirigidas a cambiar el modo en que se presta atención a la relación estresante). 
Si bien es cierto, algunos estudios han reportado una relación inversa entre la felicidad y el estrés percibido (González-Ramírez et al., 2017; Piqueras et al., 2011; Schiffrin et al., 2010), se ha investigado poco sobre los mecanismos subyacentes que pueden explicar esta relación. Es probable, que la mayor parte de las investigaciones continúen estudiando a la felicidad como una variable de resultado en lugar de analizarla como una variable predictora y explorar sus beneficios en distintos dominios de vida (Diener et al., 2018; Diener \& Tay, 2012; Lucas \& Diener, 2008).

Por tal motivo, en este estudio se plantean dos posibles rutas por las cuales la felicidad puede influir en el proceso del estrés y el afrontamiento, las cuales se detallan a continuación.

\section{Felicidad, evaluación cognitiva de desafío y afrontamiento}

Existe un creciente interés por el estudio de antecedentes que predisponen a las personas a evaluar las situaciones estresantes como desafíos (Folkman \& Moskowitz, 2000b). Algunas investigaciones sugieren que la felicidad puede ser considerada como un antecedente que influye en la evaluación cognitiva del estrés. Por ejemplo, Lyubomirsky y Tucker (1998) realizaron tres estudios que aportan evidencia a favor de la hipótesis que las personas felices perciben, interpretan y piensan acerca de los mismos eventos en un modo más positivo que sus pares menos felices.

Evaluar una situación estresante en términos de desafío implica que la situación es relevante y que los recursos o capacidades personales son suficientes para afrontar tales demandas ambientales (Blascovich, 2008; Tomaka et al., 1997). Por esta razón, existe un menor nivel de estrés subjetivo, mejor respuesta fisiológica, mayor rendimiento y esfuerzo percibido (Blascovich, 2008; Tomaka et al., 1993). Las evaluaciones de desafío predicen expectativas de confianza en el afrontamiento, mayor experiencia de emociones positivas y percepciones de beneficio en el desempeño en situaciones de evaluación (Skinner \& Brewer, 2002). Por lo general, la evaluación cognitiva de desafío se caracteriza por tener un tono más positivo y ejercer control sobre los eventos, lo cual favorece los esfuerzos de afrontamiento dirigidos a modificar el problema (Bjorck \& Cohen, 1993; Folkman \& Lazarus, 1980; Lazarus \& Folkman, 1986; McCrae, 1984; Peacock et al., 1993). En contraste, la evaluación de amenaza reduce las opciones de afrontamiento enfocados en el problema, dando lugar a esfuerzos de afrontamiento enfocados en la emoción cada vez más primitivos, desesperados y regresivos (Lazarus \& Folkman, 1986).

\section{Felicidad, emociones positivas y afrontamiento}

Las personas felices e infelices experimentan similar cantidad de eventos de vida, tanto positivos como negativos (Diener \& Seligman, 2002; Lyubomirsky \& Tucker, 1998); sin embargo, los primeros se distinguen por experimentar emociones positivas de moderada intensidad la mayor parte del tiempo (Diener et al., 1991; Diener \& Seligman, 2002). Esta mayor experiencia de emociones positivas tendría un importante papel en el afrontamiento del estrés (Lyubomirsky et al., 2005; Steptoe et al., 2008). Los estudios desarrollados por Folkman acerca del estado de ánimo y el afrontamiento en cuidadores de pacientes con $\mathrm{VIH}$ demostraron que el afecto positivo puede coexistir en períodos de tiempo estresantes, a menudo con sorprendente frecuencia, y que el afecto positivo está relacionado consistentemente a la reevaluación cognitiva, el afrontamiento enfocado en el problema y la infusión de significado positivo a eventos ordinarios (Folkman \& Moskowitz, 2000a, 2000b).

Estos hallazgos abrieron importantes cuestiones acerca del rol adaptativo de las emociones positivas en medio del estrés. De hecho, Folkman (2008) propuso una revisión del 
modelo transaccional del estrés y el afrontamiento e introdujo el afrontamiento enfocado en el significado como un tipo general de estrategias de afrontamiento asociadas a la generación y mantención de emociones positivas en el proceso del estrés.

La teoría de ampliación y construcción de emociones positivas de Fredrickson (2001) ofrece otra interesante explicación. Esta sostiene que las emociones positivas amplían momentáneamente los modos habituales de pensar y actuar de las personas, y construyen recursos físicos, intelectuales, sociales y psicológicos que funcionan como reservas que pueden utilizarse a largo plazo, incluso bajo diferentes estados afectivos, para manejar futuras amenazas. Lo cual implica un valor adaptativo indirecto y a largo plazo.

Esta ampliación mental, que permite el pensamiento creativo y flexible, facilita el afrontamiento y ayuda a regular las experiencias emocionales negativas de las situaciones estresantes (Fredrickson \& Joiner, 2002; Fredrickson et al., 2003; Pavani et al., 2015). Fredrickson y Joiner (2002) reportaron que las emociones positivas predicen tanto un afrontamiento de mentalidad abierta como la experiencia de nuevas emociones positivas. Las personas que experimentan emociones positivas en medio de situaciones estresantes traumáticas o cotidianas encuentran mayor significado positivo de los eventos adversos (Fredrickson et al., 2003; Tugade \& Fredrickson, 2004).

\section{El presente estudio}

De acuerdo con la revisión de la literatura, se puede señalar que el conocimiento sobre la influencia de la felicidad en el proceso del estrés y el afrontamiento es insuficiente. Existe la necesidad de producir más investigaciones que puedan dilucidar los mecanismos por los cuales la felicidad puede ayudar a las personas a manejar de forma efectiva y adaptativa las dificultades y obstáculos de la vida. De esta manera, al considerar que se sabe poco acerca de cómo la felicidad puede provocar resultados beneficiosos en distintos dominios de vida, este estudio pretende esclarecer dos posibles rutas por las cuales la felicidad puede promover un funcionamiento efectivo en las personas.

Sobre la base de lo expuesto, el objetivo principal fue determinar la influencia de la felicidad, por medio de la evaluación cognitiva y las emociones positivas, en el afrontamiento en estudiantes universitarios de Lima Metropolitana. Las hipótesis fueron: (a) la felicidad influye, por medio de la evaluación cognitiva de desafío, en la utilización de estrategias de afrontamiento adaptativas; (b) la felicidad influye, por medio de la experiencia de emociones positivas, en la utilización de estrategias de afrontamiento adaptativas.

\section{Método}

\section{Participantes}

Se evaluó a 352 estudiantes universitarios, 247 mujeres (70.2\%) y 105 hombres (29.8\%), con edades comprendidas entre 19 y 35 años $(M=19.5 ; D T=3.04)$, pertenecientes a distintas carreras profesionales de una universidad privada de Lima Metropolitana. El muestreo utilizado fue no probabilístico de tipo intencionado.

\section{Instrumentos}

Felicidad. Para medir la felicidad se utilizó la Escala de Felicidad de Lima (EFL; Alarcón, 2006). La EFL posee cuatro dimensiones: ausencia de sufrimiento profundo (ítem 11: "La mayoría del tiempo me siento feliz"), satisfacción con la vida (ítem 4: "Estoy satisfecho con mi vida"), realización personal (ítem 24: "Me considero una persona realizada") y alegría de vivir (ítem 12: "Es maravilloso vivir"). Está compuesta por 27 ítems con una 
escala de respuesta tipo Likert de 5 puntos, en donde 1 = "totalmente en desacuerdo" y 5 = "totalmente de acuerdo". En el presente estudio se obtuvieron buenos índices de fiabilidad para cada una de las dimensiones: ausencia de sufrimiento profundo ( $\alpha=.89$; $\omega=.89)$, satisfacción con la vida $(\alpha=.83 ; \omega=.83)$, realización personal $(\alpha=.76 ; \omega=.77)$ y alegría de vivir $(\alpha=.75 ; \omega=.75)$.

Emociones positivas y negativas. Se empleó la Escala de Afecto Positivo y Negativo forma corta (I-PANAS-SF; Thompson, 2007). Esta escala está compuesta por 10 ítems que miden el grado en el cual se experimenta afecto positivo (Ítem 7: "Decidido/a") y afecto negativo (Ítem 6: "Nervioso/a"). Los participantes debían de responder a los ítems utilizando una escala de respuesta tipo Likert de 5 puntos, en donde $1=$ "nunca" y 5 = "siempre". Se utilizó la versión validada en el contexto peruano por Gargurevich (2010). En este estudio las escalas de afecto positivo $(\alpha=.77 ; \omega=.77)$ y afecto negativo $(\alpha=.76 ; \omega$ $=.77$ ) obtuvieron adecuados índices de fiabilidad.

Evaluación cognitiva. Se utilizaron seis ítems que estiman el grado de importancia, control, eficacia, estrés, amenaza y desafío que evoca una determinada situación estresante (Chang, 1998). Se pidió a los estudiantes que valoraran una situación estresante que les hubiera sucedido en el último mes y que utilizaran una escala de respuesta tipo Likert de 10 puntos. En este estudio se encontraron aceptables coeficientes de fiabilidad para las dimensiones: evaluación cognitiva de amenaza $(\alpha=.67 ; \omega=.68)$ y evaluación cognitiva de desafío $(\alpha=.61 ; \omega=.64)$.

Estrategias de afrontamiento. Se emplearon dos instrumentos. Por un lado, el Inventario de Estrategias de Afrontamiento (CSl; Tobin et al., 1989; adaptación de Cano et al., 2007), que evalúa la utilización de ocho estrategias de afrontamiento centradas en el problema y en la emoción tanto adaptativas como desadaptativas. El CSI está compuesto por 40 ítems con una escala de respuesta tipo Likert de 5 puntos. En este estudio se obtuvieron buenos índices de fiabilidad para resolución de problemas $(\alpha=.82 ; \omega=.82)$, reestructuración cognitiva $(\alpha=.80 ; \omega=.80)$, apoyo social $(\alpha=.84 ; \omega=.85)$, expresión emocional $(\alpha=.83 ; \omega=.83)$, evitación de problemas $(\alpha=.69 ; \omega=.70)$, pensamiento desiderativo $(\alpha=.79 ; \omega=.79)$, retirada social $(\alpha=.74 ; \omega=.75)$ y autocrítica $(\alpha=.87 ; \omega$ $=.87$ ). Por otra parte, se utilizó la Escala de Significado Positivo (Fredrickson et al., 2003), compuesta por 5 ítems que miden el grado en el cual las personas encuentran significado positivo de sus problemas o estresores actuales. De igual manera, para esta escala se encontraron buenos niveles de fiabilidad $(\alpha=.85 ; \omega=.85)$.

\section{Procedimiento}

Se solicitó autorización a los directivos de la Universidad para llevar a cabo el estudio. La recolección de los datos se llevó a cabo durante los horarios de clase en una sola sesión. Se informó a los participantes sobre el objetivo del estudio y se les solicitó su participación, señalando el carácter voluntario, anónimo y confidencial del mismo. Los estudiantes que aceptaron participar firmaron el consentimiento informado, completaron una ficha sociodemográfica y los instrumentos anteriormente descritos. Además, se brindaron instrucciones verbales sobre la forma de respuesta a los instrumentos.

\section{Análisis de datos}

El análisis de los datos se llevó a cabo con el programa STATA 14 para Windows y tuvo dos niveles. Primero, se realizaron estadísticos descriptivos y correlaciones $r$ de Pearson entre las variables de estudio. En el caso de las correlaciones, valores $r \geq .20, r \geq .50$ y $r \geq$ .80 representan una correlación mínima, moderada y fuerte, respectivamente (Ferguson, 
2009). Posteriormente, se realizaron modelos de ecuaciones estructurales para someter a prueba las hipótesis planteadas. En este caso, primero se examinó el modelo de medida de los instrumentos utilizados y luego el ajuste del modelo estructural (Kline, 2005). Se empleó el método de máxima verosimilitud ajustado debido a la falta de normalidad de los ítems (Satorra \& Bentler, 1994; SB- $\chi^{2}$ ). Se utilizaron como índices de bondad de ajuste: Root Mean Square Error Approximation (RMSEA), Comparative Fit Index (CFI), TuckerLewis Index (TLI) y Standardized Root Mean Square Residuals (SRMR). Un buen ajuste fue valorado por: RMSEA $\leq .06, \mathrm{TLI}>.90, \mathrm{CFI}>.90$ y SRMR $\leq .08$ (Lei \& Wu, 2007).

\section{Resultados}

En la Tabla 1 se presentan los estadísticos descriptivos y la matriz de correlaciones entre las variables de estudio. Los resultados muestran que la felicidad correlaciona positiva y significativamente con la evaluación cognitiva de desafío y las emociones positivas y, a su vez, con la utilización de estrategias de afrontamiento adaptativas como resolución de problemas, reestructuración cognitiva y encuentro de significado positivo.

Por otro lado, antes de realizar el análisis estructural de los modelos propuestos se evaluó el modelo de medida de los instrumentos utilizados. En general, los resultados fueron satisfactorios. En el caso de la EFL el ajuste fue óptimo, SB- $\chi^{2}=546.579(g l=293), p<$ $.001, \mathrm{RMSEA}=.050, \mathrm{CFI}=.913, \mathrm{TLI}=.904, \mathrm{SRMR}=.055$. Las saturaciones factoriales $(\lambda)$ fueron adecuadas en ausencia de sufrimiento profundo (entre .531 y .772), satisfacción con la vida (entre .562 y .752), realización personal (entre .524 y .648) y alegría de vivir (entre .553 y .747). En el I-SPANAS-SF se encontró un ajuste aceptable, SB- $\chi^{2}=880.511(\mathrm{gl}$ $=45), p<.001, \mathrm{RMSEA}=.061, \mathrm{CFI}=.941, \mathrm{TLI}=.925, \mathrm{SRMR}=.071$. Las $\lambda$ fueron adecuadas para afecto positivo (entre .526 y .712) y afecto negativo (entre .449 y .737).

En cuanto a la Evaluación Cognitiva, se encontró un ajuste aceptable, SB- $\chi^{2}=32.785 \mathrm{gl}=$ 9), $p<.001, \mathrm{RMSEA}=.087, \mathrm{CFI}=.922, \mathrm{TLI}=.869, \mathrm{SRMR}=.072$. Las $\lambda$ fueron adecuadas para evaluación cognitiva de amenaza (entre 633 y .659) y evaluación cognitiva de desafío (entre 327 y .799). En tanto que, en el CSI se encontró un ajuste óptimo, SB- $\chi^{2}$ $=893.991(g l=601), p<.001$, RMSEA $=.037, \mathrm{CFI}=.930, \mathrm{TLI}=.923, \mathrm{SRMR}=.057$. Las $\lambda$ fueron significativas para resolución de problemas (entre .629 y .749), reestructuración cognitiva (entre .579 y .752), apoyo social (entre .464 y .885), expresión emocional (entre .626 y .814), evitación de problemas (entre .584 y .745), pensamiento desiderativo (entre .488 y .737), retirada social (entre .437 y .758) y autocrítica (entre .675 y .826). Asimismo, en la Escala de Significado Positivo se halló un ajuste aceptable, SB- $\chi^{2}=26.344(g l=5)$, $p<.001, \mathrm{RMSEA}=.110, \mathrm{CFI}=.966, \mathrm{TLI}=.933, \mathrm{SRMR}=.034$. Las $\lambda$ fueron significativas $\mathrm{y}$ fluctuaron entre .646 y .807.

Finalmente, para someter a prueba las hipótesis planteadas se realizaron modelos de ecuaciones estructurales para cada una de las rutas por las cuales la felicidad puede influir en el proceso del estrés y el afrontamiento. Esto es, a través de la evaluación cognitiva de desafío y la experiencia de emociones positivas, respectivamente.

En la Figura 1 se observa que el modelo propuesto sobre la influencia de la felicidad, por medio de la evaluación cognitiva de desafío, en el proceso del estrés y el afrontamiento obtuvo un buen ajuste, SB- $\chi^{2}=76.073(g l=32), p<.001$, RMSEA $=.063, \mathrm{CFI}=.962$, TLI $=.946, \mathrm{SRMR}=.046$. 


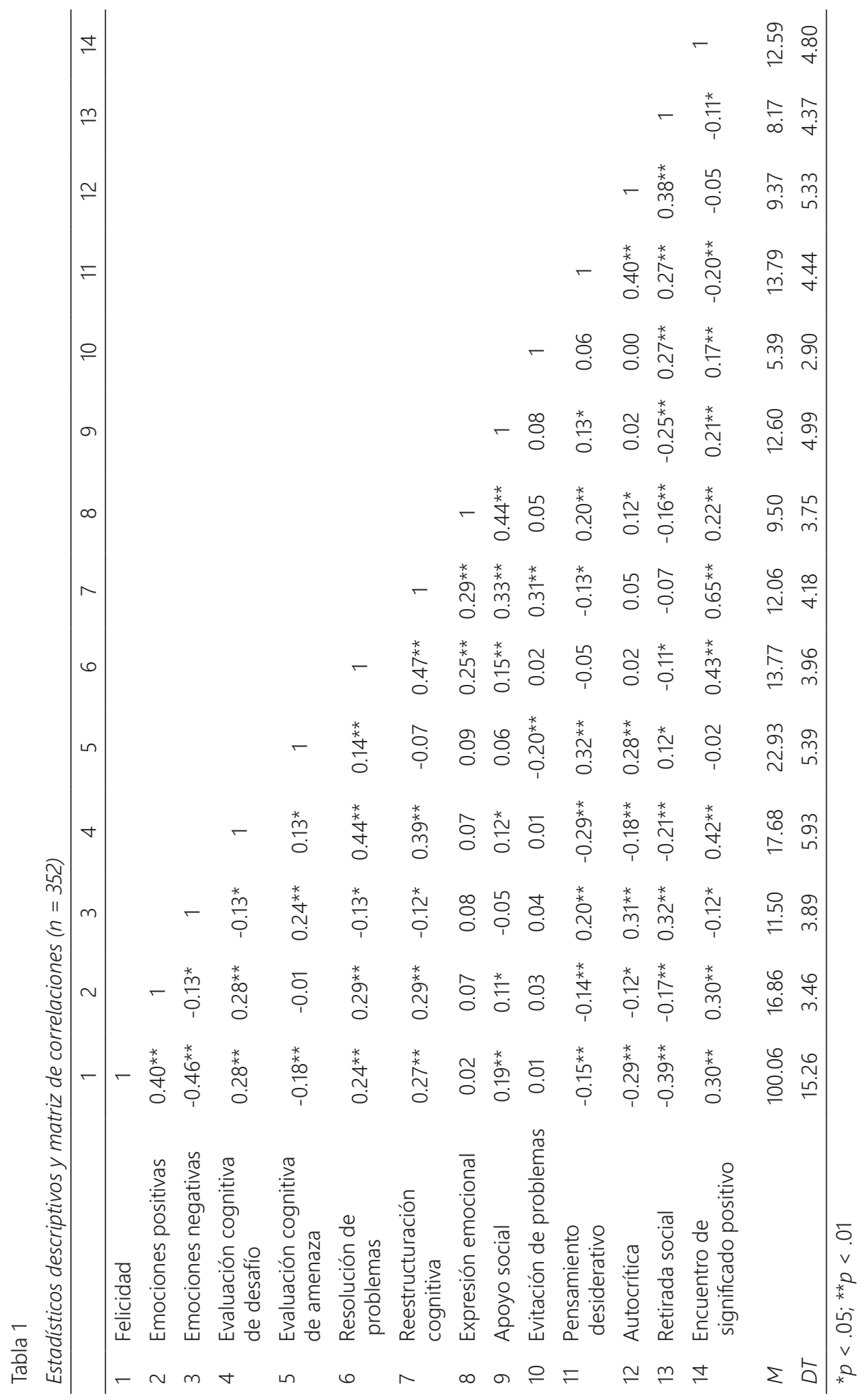

Actualidades en Psicología, 35(130), 2021, 35-48 


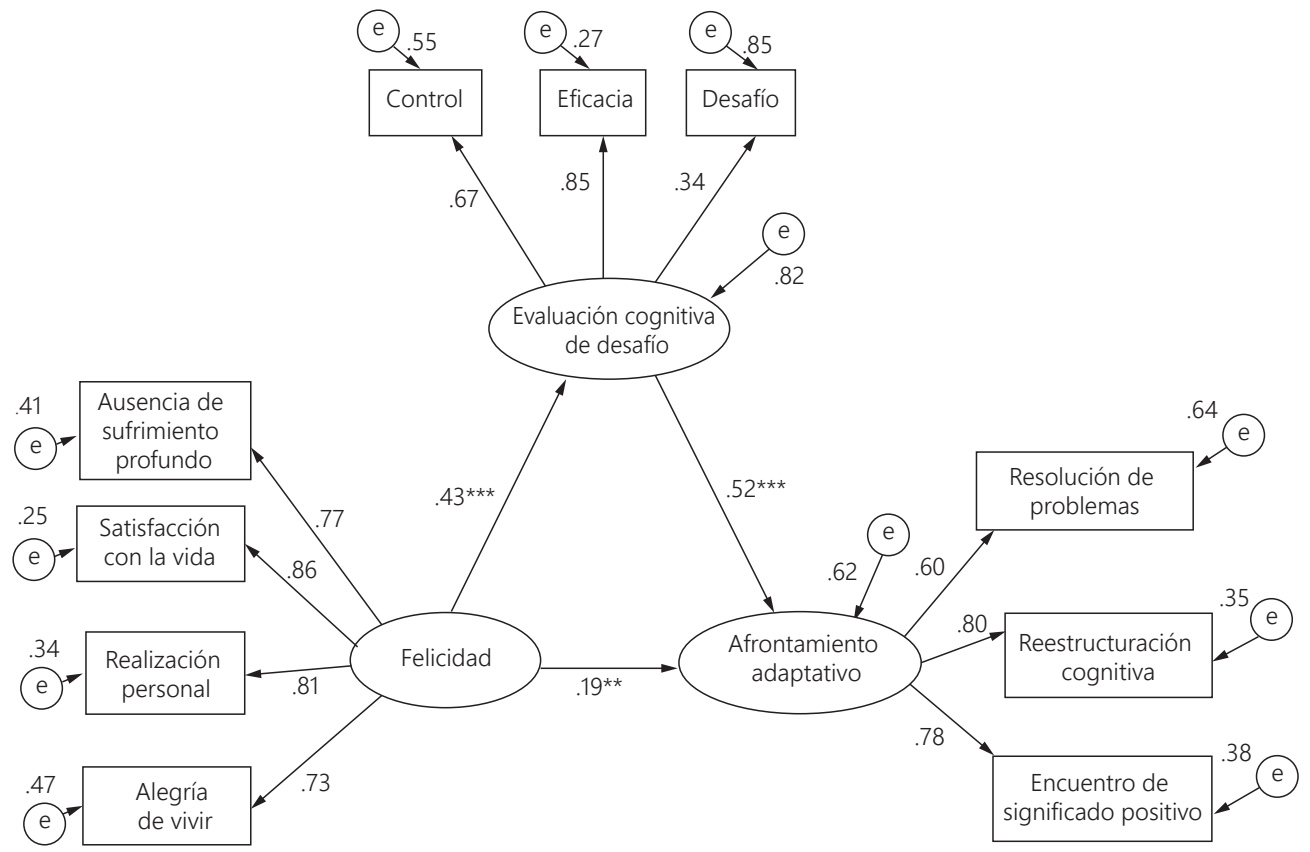

Figura 1. Modelo estructural sobre la influencia de la felicidad por medio de la evaluación cognitiva de desafío en el afrontamiento adaptativo $(n=352)$. Se muestran los coeficientes estandarizados. ${ }^{* *} p<.01 ; * * * p .001$

En la Tabla 2 se presentan los efectos directos, indirectos y totales de este primer modelo. Se observa que la felicidad tiene un efecto directo y significativo sobre la evaluación cognitiva de desafío y el afrontamiento adaptativo. De igual manera, la evaluación cognitiva de desafío media parcialmente esta relación.

En la Figura 2 se aprecia que el modelo propuesto sobre la influencia de la felicidad, por medio de las emociones positivas, en el proceso del estrés y el afrontamiento obtuvo un excelente ajuste, $\mathrm{SB}-\chi^{2}=81.718(g l=51), p<.01, \mathrm{RMSEA}=.041, \mathrm{CFI}=.977, \mathrm{TLI}=.970$, $\mathrm{SRMR}=.032$.

En la Tabla 2 se presentan los efectos directos, indirectos y totales de este segundo modelo. Se observa que la felicidad tiene un efecto directo y significativo sobre las emociones positivas y el afrontamiento adaptativo. Así mismo, las emociones positivas median parcialmente esta relación. 
Tabla 2

Resultados de los SEM: Efectos directos, indirectos y totales $(n=352)$

\begin{tabular}{|c|c|c|c|c|c|c|}
\hline & $\mathrm{B}$ & $\mathrm{EE}$ & z & $p$ & IC $95 \%$ B & $\mathrm{BE}$ \\
\hline \multicolumn{7}{|c|}{ Primer modelo: felicidad, evaluación cognitiva de desafío y afrontamiento } \\
\hline \multicolumn{7}{|l|}{ Efectos directos } \\
\hline Felicidad & .081 & .032 & 2.51 & .012 & $.018 ; .145$ & .186 \\
\hline Evaluación cognitiva de desafío & .712 & .128 & 5.59 & $<.001$ & $.462 ; .962$ & .517 \\
\hline \multicolumn{7}{|l|}{ Efecto indirecto } \\
\hline $\begin{array}{l}\text { Vía felicidad y evaluación } \\
\text { cognitiva de desafío }\end{array}$ & .096 & .023 & 4.20 & $<.001$ & $.051 ; .141$ & .220 \\
\hline \multicolumn{7}{|l|}{ Efecto total } \\
\hline Felicidad & .177 & .035 & 5.14 & $<.001$ & $.110 ; .245$ & .406 \\
\hline \multicolumn{7}{|c|}{ Segundo modelo: felicidad, emociones positivas y afrontamiento } \\
\hline \multicolumn{7}{|l|}{ Efectos directos } \\
\hline Felicidad & .102 & .036 & 2.87 & .004 & $.033 ; .173$ & .244 \\
\hline Emociones positivas & 1.056 & .277 & 3.81 & $<.001$ & $.512 ; 1.599$ & .314 \\
\hline \multicolumn{7}{|l|}{ Efectos indirectos } \\
\hline Vía Felicidad y emociones positivas & .067 & .021 & 3.22 & .001 & $.026 ; .107$ & .159 \\
\hline \multicolumn{7}{|l|}{ Efecto total } \\
\hline Felicidad & .169 & .035 & 4.88 & $<.001$ & $.101 ; .237$ & .403 \\
\hline
\end{tabular}

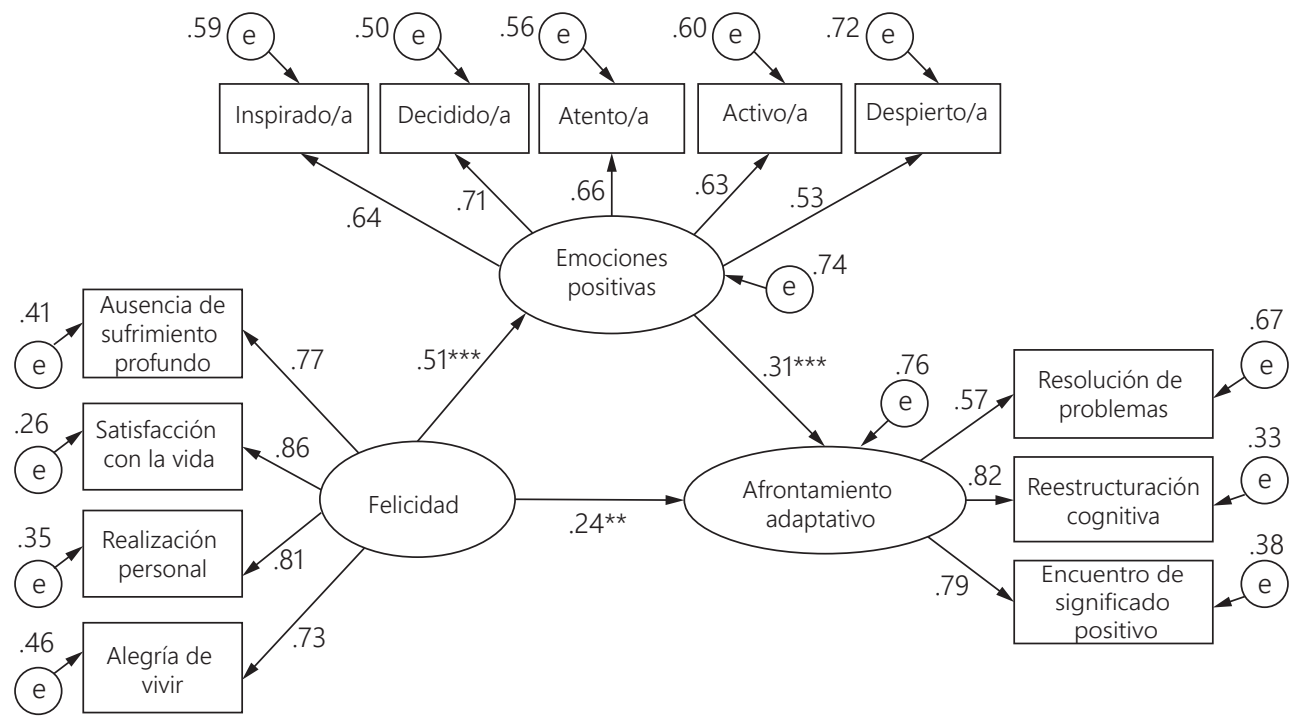

Figura 2. Modelo estructural sobre la influencia de la felicidad por medio de las emociones positivas en el afrontamiento adaptativo $(n=352)$. Se muestran los coeficientes estandarizados. ${ }^{* *} p<.01 ;{ }^{* \star *} p<.001$ 


\section{Discusión}

La presente investigación tuvo como objetivo principal determinar la influencia de la felicidad, por medio de la evaluación cognitiva y las emociones positivas, en el afrontamiento en estudiantes universitarios de Lima Metropolitana. Los resultados encontrados confirman las hipótesis planteadas y muestran que la felicidad influye en la evaluación cognitiva de desafío y la experiencia de emociones positivas, las cuales a su vez predicen la utilización de estrategias de afrontamiento adaptativas. Por ahora, este es uno de los primeros estudios que intenta comprender los mecanismos que subyacen a la asociación entre felicidad y afrontamiento, puesto que se pone especial énfasis en el rol mediador de la evaluación cognitiva de desafío y la experiencia de emociones positivas.

En ese sentido, este estudio se adhiere a una reciente línea de investigación dentro de la psicología positiva, enfocada en el estudio de los beneficios o resultados positivos que la felicidad puede producir en múltiples dominios de vida (Diener et al., 2018; Diener \& Chan, 2011; Diener \& Tay, 2012; Lucas \& Diener, 2008; Lyubomirsky et al., 2005) y asume a esta como una variable predictora en lugar de estudiarla como una variable de resultado (Abbe et al., 2003). Es decir, en lugar de preguntar qué variables hacen felices a las personas, se interesa por descubrir cómo las personas felices piensan, evalúan, deciden y responden a los eventos en comparación con las personas menos felices (Abbe et al., 2003; Lyubomirsky, 2001; Lyubomirsky \& Tucker, 1998).

Esta investigación aporta evidencia a favor de la hipótesis que las personas felices afrontan mejor las situaciones estresantes (Lyubomirsky et al., 2005; Steptoe et al., 2008). El primer modelo muestra que las personas felices tienden a evaluar las situaciones estresantes más en términos de desafío que amenaza (Lyubomirsky \& Tucker, 1998) y, en consecuencia, utilizan estrategias de afrontamiento adaptativas para manejar el estrés (Bjorck \& Cohen, 1993; Folkman \& Lazarus, 1980; McCrae, 1984; Peacock et al., 1993). El segundo modelo muestra que las personas felices suelen experimentar una mayor cantidad de emociones positivas (Cohn et al., 2009; Diener et al., 1991; Diener \& Seligman, 2002; Pavot et al., 1990; Seidlitz \& Diener, 1993) y, debido a esto, emplean estrategias de afrontamiento adaptativas para manejar las demandas propias del estrés (Fredrickson \& Joiner, 2002; Fredrickson et al., 2003; Moskowitz et al., 1996; Pavani et al., 2015; Steptoe et al., 2008; Tugade \& Fredrickson, 2004). Por consiguiente, los individuos felices logran afrontar efectivamente los eventos estresantes, situándose en una posición favorable hacia la consecución de sus objetivos y el logro de resultados exitosos en sus vidas.

Los hallazgos expuestos en este trabajo deben evaluarse con cierta precaución, en la medida en que existen algunas limitaciones que dificultan la generalización de los resultados. Por ejemplo, la muestra estuvo conformada por estudiantes universitarios mayoritariamente mujeres de una universidad privada de Lima Metropolitana y se utilizó un muestreo no probabilístico de tipo intencionado.

No obstante, sin dejar de lado estas limitaciones, este estudio ofrece importantes aportes hacia un mayor conocimiento del papel de la felicidad en el proceso del estrés y el afrontamiento. Se esclarecen dos posibles rutas por las cuales las personas felices logran manejar efectivamente las dificultades o eventos de vida que se les presentan, y a su vez, posibilita acercarse más a la comprensión de los mecanismos por los cuales la felicidad puede contribuir a obtener resultados positivos en la vida de las personas. Este es uno de los desafíos más importantes en la investigación actual sobre la felicidad (Diener et al., 2018). 
Por lo tanto, futuras investigaciones deberán tratar de superar las limitaciones encontradas aquí y profundizar en el estudio de los beneficios de la felicidad en la vida de las personas. Los resultados encontrados en este trabajo pueden utilizarse como puntos de partida para la formulación de hipótesis de investigación sobre el papel de la felicidad en el proceso del estrés y el afrontamiento en otros contextos y grupos de individuos.

\section{Referencias}

Abbe, A., Tkach, C., \& Lyubomirsky, S. (2003). The art of living by dispositionally happy people. Journal of Happiness Studies, 4(4), 385-404. https://doi.org/10.1023/ B:JOHS.0000005769.54611.3C

Alarcón, R. (2006). Desarrollo de una escala factorial para medir la felicidad. Revista Interamericana de Psicología, 40(1), 99-106. http://www.redalyc.org/articulo. oa? id $=28440110$

Bjorck, J., \& Cohen, L. (1993). Coping with threats, losses, and challenges. Journal of Social and Clinical Psychology, 12(1), 56-72. https://doi.org/10.1521/jscp.1993.12.1.56

Blascovich, J. (2008). Challenge and threat. In A. J. Elliot (Ed.), Handbook of approach and avoidance motivation (pp. 431-445). Psychology Press.

Cano, F., Rodríguez, L., \& García, J. (2007). Adaptación española del Inventario de Estrategias de Afrontamiento. Actas Españolas de Psiquiatría, 35(1), 29-39. https:// dialnet.unirioja.es/servlet/articulo?codigo $=2249538$

Chang, E. (1998). Dispositional optimism and primary and secondary appraisal of a stressor: Controlling for confounding influences and relations to coping and psychological and physical adjustment. Journal of Personality and Social Psychology, 74(4), 11091120. http://doi.org/10.1037/0022-3514.74.4.1109

Cohn, M., Fredrickson, B., Brown, S., Mikels, J., \& Conway, A. (2009). Happiness unpacked: Positive emotions increase life satisfaction by building resilience. Emotion, 9(3), 361-368. http://doi.org/10.1037/a0015952

Diener, E. (2000). Subjective well-being. The science of happiness and a proposal for a national index. American Psychologist, 55(1), 34-43. http://doi.org/10.1037/0003-066X.55.1.34

Diener, E., \& Chan, M. (2011). Happy people live longer: Subjective well-being contributes to health and longevity. Applied Psychology: Health and Well-Being, 3(1), 1-43. https://doi.org/10.1111/j.1758-0854.2010.01045.x

Diener, E., Oishi, S., \& Tay, L. (2018). Advances in subjective well-being research. Nature Human Behaviour, 2, 253-260. https://doi.org/10.1038/s41562-018-0307-6

Diener, E., Sandvik, E., \& Pavot, W. (1991). Happiness is the frequency, not the intensity, of positive versus negative affect. In F. Strack, M. Argyle, \& N. Schwarz (Eds.), Subjective Well-being: An Interdisciplinary Perspective (pp. 119-139). Pergamon Press.

Diener, E., \& Seligman, M. (2002). Very happy people. Psychological Science, 13(1), 81-84. https://doi.org/10.1111/1467-9280.00415

Diener, E., \& Tay, L. (2012). A scientific review of the remarkable benefits of happiness for successful and healthy living. Report of the Well-Being Working Group, Royal Government of Bhutan. Report to the United Nations General Assembly, WellBeing, and Happiness: A New Development Paradigm. 
Ferguson, C. J. (2009). An effect size primer: A guide for clinicians and researchers. Professional Psychology: Research and Practice, 40(5), 532-538. https://doi. org/10.1037/a0015808

Folkman, S. (2008). The case for positive emotions in the stress process. Anxiety, Stress \& Coping, 21(1), 3-14. http://doi.org/10.1080/10615800701740457

Folkman, S., \& Lazarus, R. (1980). An analysis of coping in a middle-aged community sample. Journal of Health and Social Behavior, 21(3), 219-239. http://doi. org/10.2307/2136617

Folkman, S., \& Moskowitz, J. (2000a). Positive affect and the other side of coping. American Psychologist, 55(6), 647-654. http://doi.org/10.1037/0003-066X.55.6.647

Folkman, S., \& Moskowitz, J. (2000b). Stress, positive emotion, and coping. Current Directions in Psychological Science, 9(4), 115-118. https://doi.org/10.1111/14678721.00073

Fredrickson, B. (2001). The role of positive emotions in positive psychology. The broadenand-build theory of positive emotions. American Psychologist, 56(3), 218-226. http://doi.org/10.1037/0003-066X.56.3.218

Fredrickson, B., \& Joiner, T. (2002). Positive emotions trigger upward spirals toward emotional well-being. Psychological Science, 13(2), 172-175. http://doi. org/10.1111/1467-9280.00431

Fredrickson, B., Tugade, M., Waugh, C., \& Larkin, G. (2003). What good are positive emotions in crises? A prospective study of resilience and emotions following the terrorist attacks on the United States on September 11th, 2001. Journal of Personality and Social Psychology, 84(2), 365-376. http://doi.org/10.1037/0022-3514.84.2.365

Gargurevich, R. (2010). Propiedades psicométricas de la versión internacional de la Escala de Afecto Positivo y Negativo-forma corta (I- Spanas SF) en estudiantes universitarios. Persona, (13), 31-42. https://doi.org/10.26439/persona2010. n013.263

González-Ramírez, M., Landero-Hernández, R., Quezada-Berumen, L., \& Ibarra-González, L. (2017). Stressful situations affecting the perception of happiness: Love as a stressor. Ansiedad y Estrés, 23(1), 1-5. https://doi.org/10.1016/j.anyes.2016.11.002

Kline, R. B. (2005). Principles and practice of structural equation modeling. Guilford Press.

Lazarus, R., \& Folkman, S. (1986). Estrés y Procesos Cognitivos. Martínez Roca.

Lei, P. W., \& Wu, Q. (2007). Introduction to structural equation modeling: issues and practical considerations. Educational Measurement: Issues and Practice, 26(3), 3343. http://dx.doi.org/10.1111/j.1745-39z92.2007.00099.x

Lucas, R., \& Diener, E. (2008). Subjective well-being. In M. Lewis, J. Haviland-Jones, \& L. Feldman (Eds.), The Handbook of Emotions (pp. 471-484). The Guilford Press.

Lyubomirsky, S. (2001). Why are some people happier than others? American Psychologist, 56(3), 239-249. https://doi.org/10.1037/0003-066X.56.3.239

Lyubomirsky, S., King, L., \& Diener, E. (2005). The benefits of frequent positive affect: does happiness lead to success? Psychological Bulletin, 137(6), 803-855. http:// doi.org/10.1037/0033-2909.131.6.803 
Lyubomirsky, S., \& Tucker, K. (1998). Implications of individual differences in subjective happiness for perceiving, interpreting, and thinking about life events. Motivation and Emotion, 22(2), 155-186. https://doi.org/10.1023/A:1021396422190

McCrae, R. (1984). Situational determinants of coping responses: Loss, threat, and challenge. Journal of Personality and Social Psychology, 46(4), 919-928. http://doi. org/10.1037/0022-3514.46.4.919

Moskowitz, J., Folkman, S., Collette, L., \& Vittinghoff, E. (1996). Coping and mood during AIDS-related caregiving and bereavement. Annals of Behavioral Medicine, 18(1), 49-57. https://doi.org/10.1007/BF02903939

Pavani, J. B., Le Vigouroux, S., Kop, J. L., Congard, A., \& Dauvier, B. (2015). Affect and affect regulation strategies reciprocally influence each other in daily life: The case of positive reappraisal, problem-focused coping, appreciation, and rumination. Journal of Happiness Studies, 17(5), 2077-2095. https://doi.org/10.1007/s10902015-9686-9

Pavot, W., Diener, E., \& Fujita, F. (1990). Extraversion and happiness. Personality and Individual Differences, 11(12), 1299-1306. http://doi.org/10.1016/0191-8869(90)90157-M

Peacock, E., Wong, P., \& Reker, G. (1993). Relations between appraisals and coping schemas: Support for the congruence model. Canadian Journal of Behavioral Science, 25(1), 64-80. http://doi.org/10.1037/h0078787

Piqueras, J. A., Kuhne, W., Vera-Villarroel, P., Van Straten, A., \& Cuijpers, P. (2011). Happiness and health behaviours in Chilean college students: A cross-sectional survey. BMC Public Health, 11(1), 443-453. https://doi.org/10.1186/1471-2458-11-443

Satorra, A., \& Bentler, P. M. (1994). Corrections to test statistics and standard errors incovariance structure analysis. In A. von Eye, \& C. C. Clogg (Eds.), Latent variables analysis: Applications for developmental research (pp. 399-419). Sage Publications.

Schiffrin, H., Rezendes, D., \& Nelson, S. (2010). Stressed and happy? Investigating the relationship between happiness and perceived stress. Journal of Happiness Studies, 11(1), 33-39. http://doi.org/10.1007/s10902-008-9104-7

Seidlitz, L., \& Diener, E. (1993). Memory for positive versus negative life events: Theories for the differences between happy and unhappy persons. Journal of Personality and Social Psychology, 64(4), 654-664. http://doi.org/10.1037/0022-3514.64.4.654

Skinner, N., \& Brewer, N. (2002). The dynamics of threat and challenge appraisals prior to stressful achievement events. Journal of Personality and Social Psychology, 83(3), 678-692. http:// doi.org/10.1037/0022-3514.83.3.678

Steptoe, A., O'Donnell, K., Marmot, M., \& Wardle, J. (2008). Positive affect and psychosocial processes related to health. British Journal of Psychology, 99(2), 211-227. https:// doi.org/10.1111/j.2044-8295.2008.tb00474.x

Thompson, E. R. (2007). Development and validation of an internationally reliable ShortForm of the Positive and Negative Affect Schedule (PANAS). Journal of CrossCultural Psychology, 38(2), 227-242. https://doi.org/10.1177/0022022106297301

Tobin, D., Holroyd, K., Reynolds, R., \& Wigal, J. (1989). The hierarchical factor structure of the Coping Strategies Inventory. Cognitive Therapy and Research, 13(4), 343-361. https://doi.org/10.1007/BF01173478 
Tomaka, J., Blascovich, J., Kelsey, R., \& Leitten, C. (1993). Subjective, physiological, and behavioral effects of threat and challenge appraisal. Journal of Personality and Social Psychology, 65(2), 248-260. http://doi.org/10.1037/0022-3514.65.2.248

Tomaka, J., Blascovich, J., Kibler, J., \& Ernst, J. (1997). Cognitive and physiological antecedents of threat and challenge appraisal. Journal of Personality and Social Psychology, 73(1), 63-72. http://doi.org/10.1037/0022-3514.73.1.63

Tugade, M., \& Fredrickson, B. (2004). Resilient individuals use positive emotions to bounce back from negative emotional experiences. Journal of Personality and Social Psychology, 86(2), 320-333. http://doi.org/10.1037/0022-3514.86.2.320 